\title{
Interobserver reliability is higher for assessments with 3D software-generated models than with conventional MRI images in the classification of trochlear dysplasia
}

\author{
Andreas Fuchs $^{1}$ (1) Matthias J. Feucht ${ }^{2} \cdot$ Jörg Dickschas ${ }^{3} \cdot$ Jannik Frings $^{4} \cdot$ Markus Siegel $^{1} \cdot$ Tayfun Yilmaz $^{1}$. \\ Hagen Schmal ${ }^{1} \cdot$ Kaywan Izadpanah $^{1}$
}

Received: 11 April 2021 / Accepted: 10 August 2021 / Published online: 22 August 2021

(c) The Author(s) 2021

\begin{abstract}
Purpose Trochlear dysplasia is a significant risk factor for patellofemoral instability. The severity of trochlear dysplasia is commonly evaluated based on the Dejour classification in axial MRI slices. However, this often leads to heterogeneous assessments. A software to generate MRI-based 3D models of the knee was developed to ensure more standardized visualization of knee structures. The purpose of this study was to assess the intra- and interobserver agreements of 2D axial MRI slices and an MRI-based 3D software generated model in classification of trochlear dysplasia as described by Dejour.

Methods Four investigators independently assessed 38 axial MRI scans for trochlear dysplasia. Analysis was made according to Dejour's 4 grade classification as well as differentiating between 2 grades: low-grade (types A+B) and high-grade trochlear dysplasia (types C+D). Assessments were repeated following a one-week interval. The inter- and intraobserver agreement was determined using Cohen's kappa $(\kappa)$ and Fleiss kappa statistic $(\kappa)$. In addition, the proportion of observed agreement (po) was calculated for assessment of intraobserver agreement.

Results The assessment of the intraobserver reliability with regard to the Dejour-classification showed moderate agreement values both in the $2 \mathrm{D}(\kappa=0.59 \pm 0.08 \mathrm{SD})$ and in the $3 \mathrm{D}$ analysis $(\kappa=0.57 \pm 0.08 \mathrm{SD})$. Considering the 2 -grade classification, the $2 \mathrm{D}(\kappa=0.62 \pm 0.12 \mathrm{SD})$ and $3 \mathrm{D}$ analysis $(\kappa=0.61 \pm 0.19 \mathrm{SD})$ each showed good intraobserver matches. The analysis of the interobserver reliability also showed moderate agreement values with differences in the subgroups (2D vs. 3D). The 2D evaluation showed correspondences of $\kappa=0.48$ (Dejour) and $\kappa=0.46$ (high / low). In the assessment based on the 3D models, correspondence values of $\kappa=0.53$ (Dejour) and $\kappa=0.59$ (high / low) were documented.

Conclusion Overall, moderate-to-good agreement values were found in all groups. The analysis of the intraobserver reliability showed no relevant differences between 2 and 3D representation, but better agreement values were found in the 2-degree classification. In the analysis of interobserver reliability, better agreement values were found in the 3D compared to the $2 \mathrm{D}$ representation. The clinical relevance of this study lies in the superiority of the 3D representation in the assessment of trochlear dysplasia, which is relevant for future analytical procedures as well as surgical planning.
\end{abstract}

Level of evidence Level II.

Keywords Trochlear dysplasia $\cdot$ Patellar instability $\cdot$ Dejour $\cdot$ 3D model

\section{Introduction}

Trochlear morphology is recognized as one of the most important factors for patellar stability $[6,9,11,18,19,26]$. Trochlear dysplasia is estimated to occur in less than $2 \%$

Andreas Fuchs

andreas.fuchs@uniklinik-freiburg.de

Extended author information available on the last page of the article of the general population, whereas, $62 \%-96 \%$ of patients with patellar instability have evidence of trochlear dysplasia $[5,6,10]$. The severity of trochlear dysplasia is commonly evaluated and categorized based on the Dejour radiographic and magnetic resonance imaging (MRI) classifications [9, $12,15]$. In the past decades MRI has become the standard to assess patellofemoral instability [1, 4, 8, 20, 22, 25]. On axial MRI, trochlear dysplasia is diagnosed on the first craniocaudal image, where the complete cartilaginous trochlea can be seen. Dejour classified trochlear dysplasia into type 
A (fairly shallow trochlea), type B (flat or convex trochlea), type $\mathrm{C}$ (asymmetry of trochlear facets with a hypoplastic medial condyle), and type D (asymmetry of trochlear facets plus vertical join and cliff pattern) [5]. However, poor interand intraobserver agreement values using Dejour's 4 type classification has been reported in evaluation of axial MRI $[12,13,15,27]$ with low correlation to objective intraoperative findings [14].

Due to the complex surface of anatomy observed in trochlear dysplasia, the best possible and standardized visualization of the bony and cartilage structures is of crucial importance. Here, 3D imaging possibly offers good options. It allows to present the individual anatomy in a physical three-dimensional model and potentially eases the capture of spatial proportions, especially in cases of complex anatomy [7]. For this reason, an MRI-based 3D model of the knee was created, in order to enable an improved analysis of the complex anatomical conditions and thus a more reliable therapy planning in the future. The aim of this study was to assess the intra- and interobserver agreements of $2 \mathrm{D}$ axial magnetic resonance images and an MRI-based 3D software-generated model in classification of trochlear dysplasia and to compare the commonly used Dejour's 4-grade-classication system with a 2-grade-classification system based on Dejour's classification. It was hypothesized that there are better agreement values in $3 \mathrm{D}$ compared to $2 \mathrm{D}$ representation.

\section{Materials and methods}

A retrospective evaluation of $38 \mathrm{MRI}$ scans of 38 patients with trochlear dysplasia was performed. This retrospective study was approved by an institutional review board (Technical University Munich, ID-number: 208/10 S-KK). The selection of the MRI scans was made at random by one of the authors out of 80 patients who had undergone patellofemoral stabilization surgery within a year without reference to the extent of the trochlear dysplasia present. Patients with previous surgery on bony structures of the knee or MRI images of poor quality $(<1.5$ Tesla) were excluded. The allocation of the 38 knee joints with trochlear dysplasia according to Dejour (Consensus of all investigators after independent repeated classification of trochlea types-the maximum agreement of the independent classification of all investigators was decisive for the consensus) showed the following distribution: type A: $n=13$, type B: $n=14$, type $\mathrm{C}: n=5$, type $\mathrm{D}: n=6$. None of the patients had any history of knee surgery altering the form of the femoral trochlea prior to MRI.

Four orthopedic surgeons independently graded the trochlear shape in axial T2-weighted MRI slices of the most proximal transverse MRI where the cartilage along the entire width of the trochlea was visible. Each MRI was performed in normal clinical routine with the patient in a supine position. Due to the acquisition of patients in normal clinical routine, the MRI images were produced on different devices in different institutions. Inclusion criteria for use within this study were: MRI $\geq 1.5$ Tesla, representation of the entire trochlea, no movement artifacts and no metal artifacts. Afterwards, all four surgeons repeated the assessment and classification on MRI based 3D image-models of the same patients. Both in the assessment of axial MRI slices and in the assessment of 3D models, it was possible to switch freely between the slices or views. The standard 3D view showed both bone and cartilage tissue, but it was also possible to temporarily hide the cartilage structures. Both assessments were repeated by all surgeons after a minimum interval of one week. The order of the cases was randomized to eliminate any memory bias.

The 3D models were generated by slice-wise segmentation of the 38 MRI scans. Segmentation of bone and cartilage was initially done manually for the whole cohort in a web-based application (Fraunhofer MEVIS Knee SATORI, Version 1.0.0a). Several positions inside and outside the respective structure were manually marked until the corresponding segmentation, which was updated in real time, highlighted the correct extent. The resulting surface was postprocessed by snapping it to a subvoxel precise position based on a cubic interpolation of the image data. The results of the manual segmentations of all subjects were used to train the web application in segmentation for the different structures. The trained model was then applied to the same images for more consistent segmentation results. After segmentation (manual or automatic), the 3D models are created immediately using the web application.

The results were first analyzed with regards to the four classification types (Dejour) between the four readers. For further investigation, a differentiation in a 2-grade classification system was additionally chosen. Here, the subgroups "Low grade" dysplasia (Dejour A + B) and "High grade" dysplasia (Dejour C+D) were investigated Figs 1 and 2.

\section{Statistical analysis}

The intraobserver/intermethod agreement was determined using Cohen's kappa statistic. Interobserver agreement was assessed using Fleiss kappa statistic. The kappa statistic expresses the chance-corrected agreement. It is the (normalized) observed agreement minus the agreement expected on the basis of chance alone. The expected agreement is based on the prevalence of each grade, which was calculated from the combined ratings of all raters. A kappa value greater than 0.75 represents excellent agreement, values between 0.60 and 0.74 good, values between 0.40 and 0.59 moderate, values between 0.21 and 0.40 slight and values below 0.20 poor agreement [24]. 
Fig. 1 Segmentation of cartilage (blue) and bone (green) structures of the distal femur in T2 weighted sagittal MRI slices. *Blue line: segmentation of femoral cartilage in sagittal MRI slices for the creation of a 3D model; Green line: segmentation of femoral bone in sagittal MRI slices for the creation of a 3D model
Fig. 2 Software-generated MRIbased 3D model of the knee.

**Blue structure: representation of the femoral cartilage in a $3 \mathrm{D}$ model; Green structure: representation of the femoral bone in a 3D model; Left camera orientation: foot, Right camera orientation: head
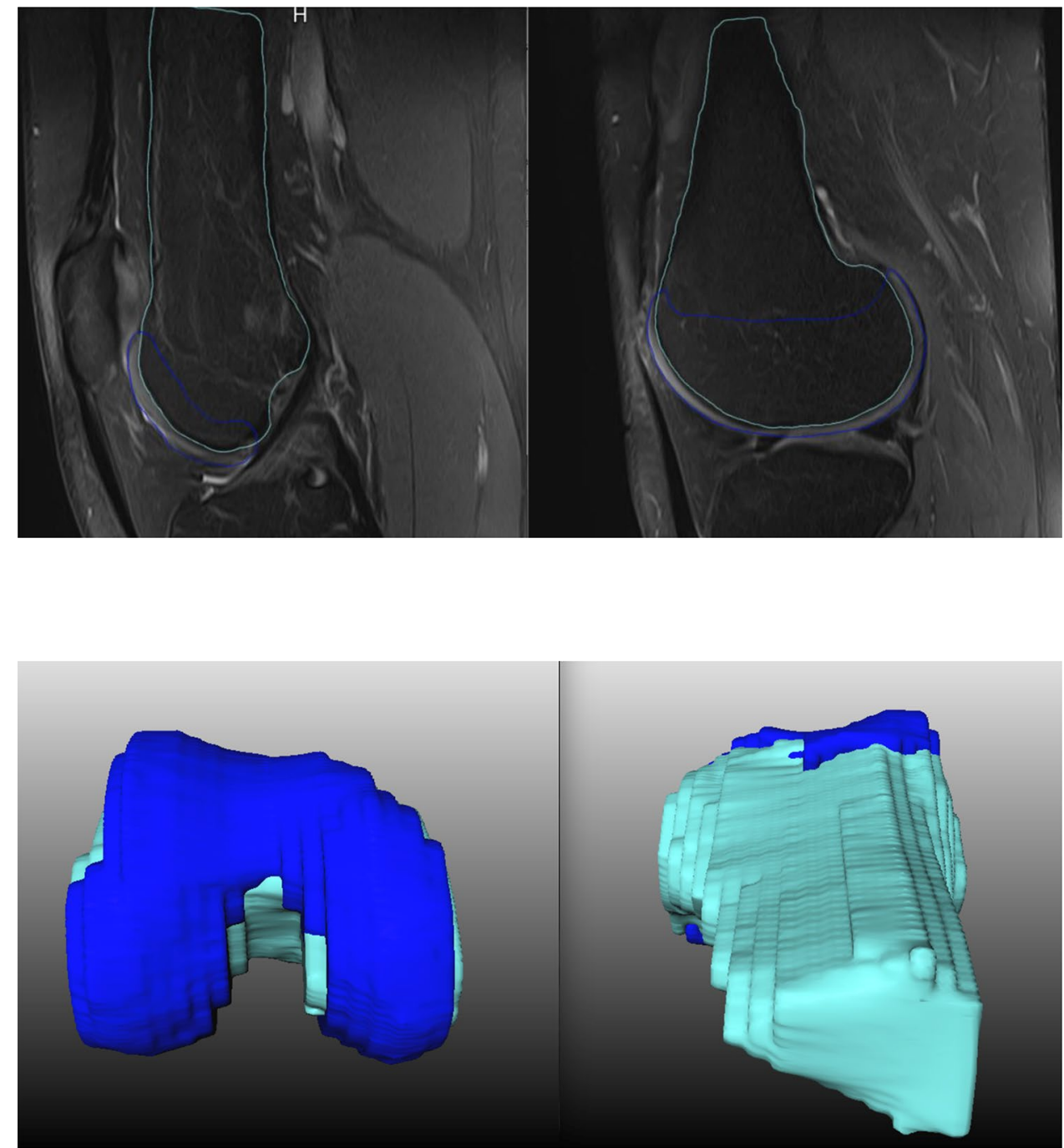

In addition, the proportion of observed agreement (po) was calculated, including $95 \%$ confidence interval (CI), for assessment of intraobserver agreement. The proportion $\mathrm{p}_{0}$ describes the percentage of agreement and is calculated from the sum of the observed absolute frequencies in the main diagonal of contingency tables divided by the total number of patients.

Three different investigations were performed separately for the 4-graded classification as well as the 2-graded classification:

1. the intraobserver agreement was investigated (agreement of ratings made at the first and second time points of assessment: for each rater, 2D and 3D, Cohens Kappa and proportion of agreement).

2. the intermethod agreement of $2 \mathrm{D}$ and $3 \mathrm{D}$ analysis was investigated (for each rater, for each time point of assessment, Cohens Kappa and proportion of agreement).
3. the interobserver agreement was investigated (a comparison of all raters for each time point of assessment, 2D and 3D, Fleiss Kappa).

Statistical analyses were carried out using IBM SPSS Statistics Version 27.0.0.0 (IBM Corp., Armonk, New York). The results of all statistical tests were interpreted in an exploratory sense.

\section{Results}

In the following, relevant results are summarized in Kappa values $(\kappa)$ and proportion of agreement $\left(\mathrm{p}_{0}\right)$. 
Table 1 Intraobserver agreement, 2-grade- and 4-grade analysis*

\begin{tabular}{|c|c|c|c|c|}
\hline \multirow[t]{2}{*}{ Rater } & \multicolumn{2}{|c|}{ 4-grade analysis (Dejour) } & \multicolumn{2}{|c|}{ 2-grade analysis (High/Low) } \\
\hline & $2 \mathrm{D}$ & 3D & $2 \mathrm{D}$ & $3 \mathrm{D}$ \\
\hline \multicolumn{5}{|c|}{ Cohen's Kappa } \\
\hline 1 & 0.59 & 0.66 & 0.58 & 0.82 \\
\hline 2 & 0.71 & 0.49 & 0.77 & 0.71 \\
\hline 3 & 0.57 & 0.54 & 0.78 & 0.51 \\
\hline 4 & 0.51 & 0.60 & 0.36 & 0.41 \\
\hline \multicolumn{5}{|c|}{ Proportion of agreement } \\
\hline 1 & $69 \%(54 ; 84)$ & $74 \%(60 ; 88$ & $81 \%(68 ; 94)$ & $92 \%(83 ; 100)$ \\
\hline 2 & $65 \%(50 ; 80)$ & $71 \%(56 ; 86)$ & $70 \%(55 ; 85)$ & $70 \%(55 ; 85)$ \\
\hline 3 & $78 \%(65 ; 91)$ & $63 \%(47 ; 79)$ & $89 \%(79 ; 99)$ & $87 \%(76 ; 98)$ \\
\hline 4 & $68 \%(53 ; 83)$ & $64 \%(49 ; 79)$ & $89 \%(79 ; 99)$ & $76 \%(62 ; 90)$ \\
\hline
\end{tabular}

*4-grade analysis, Dejour classification; 2-grade analysis, high/low grade trochlear dysplasia; 2D, 2D axial MRI scan; 3D, MRI-based 3D model; values in parenthesis represent $95 \%$ confidence interval

\section{Intraobserver agreement, 4-grade analysis}

When observers classified trochlear dysplasia into Dejour's four grades, the intraobserver agreement in 2D MRI evaluation between the first and the second reading was $65-78 \%$. The mean Kappa value (Cohens Kappa) was 0.59 ( $\mathrm{SD} \pm 0.08)$. Intraobserver agreement for classification into four grades for the two readings of the 3D models was $63-74 \%$. The mean Kappa value (Cohens Kappa) was $0.57(\mathrm{SD} \pm 0.08)$ (Table 1$)$.

\section{Intraobserver agreement, 2-grade-analysis}

The intraobserver agreement in the evaluation of the subgroups low-grade dysplasia (Dejour A + B) and high-grade dysplasia (Dejour C + D) between the first and the second reading was $70-89 \%$ in $2 \mathrm{D}$ - and $70-92 \%$ in $3 \mathrm{D}$-analysis. The mean Kappa values (Cohens Kappa) were 0.62 $(\mathrm{SD} \pm 0.12)$ for $2 \mathrm{D}$ - and $0.61(\mathrm{SD} \pm 0.19)$ for $3 \mathrm{D}$ - assessment. (Table 1).

\section{Intermethod agreement of 2D- and 3D-evaluation, 4-grade analysis}

Using the 4-grade classification according to Dejour, the agreement of $2 \mathrm{D}$ and $3 \mathrm{D}$ evaluation at the first reading was 41-57\%. At the second reading, an agreement between 41 and $59 \%$ was achieved. These findings correspond to mean Kappa-values (Cohens Kappa) of $\kappa=0.32(\mathrm{SD} \pm 0.09)$ for the first reading and $\kappa=0.35(\mathrm{SD} \pm 0.15)$ for the second reading (Table 2 ).
Table 2 Intermethod agreement, 2-grade- and 4-grade analysis**

\begin{tabular}{|c|c|c|c|c|}
\hline \multirow[t]{2}{*}{ Rater } & \multicolumn{2}{|c|}{ 4-grade analysis (Dejour) } & \multicolumn{2}{|c|}{$\begin{array}{l}\text { 2-grade analysis (High/ } \\
\text { Low) }\end{array}$} \\
\hline & $\mathrm{t} 1$ & $\mathrm{t} 2$ & $\mathrm{t} 1$ & $\mathrm{t} 2$ \\
\hline \multicolumn{5}{|c|}{ Cohen's Kappa } \\
\hline 1 & 0.41 & 0.40 & 0.76 & 0.51 \\
\hline 2 & 0.33 & 0.45 & 0.48 & 0.66 \\
\hline 3 & 0.34 & 0.42 & 0.29 & 0.35 \\
\hline 4 & 0.19 & 0.12 & 0.27 & 0.30 \\
\hline \multicolumn{5}{|c|}{ Proportion of agreement } \\
\hline 1 & $57 \%(41 ; 73)$ & $57 \%(41 ; 73)$ & $89 \%(79 ; 99)$ & $78 \%(65 ; 91)$ \\
\hline 2 & $41 \%(50 ; 80)$ & $41 \%(25 ; 57)$ & $65 \%(50 ; 80)$ & $65 \%(50 ; 80)$ \\
\hline 3 & $51 \%(65 ; 91)$ & $59 \%(43 ; 75)$ & $76 \%(62 ; 90)$ & $84 \%(72 ; 96)$ \\
\hline 4 & $51 \%(53 ; 83)$ & $57 \%(41 ; 73)$ & $65 \%(50 ; 80)$ & $68 \%(53 ; 83)$ \\
\hline
\end{tabular}

*** 4-grade analysis, Dejour classification; 2-grade analysis, high/low grade trochlear dysplasia; $\mathrm{t} 1$, time point $1 ; \mathrm{t} 2$, time point 2 ; values in parenthesis represent $95 \%$ confidence interval

\section{Intermethod agreement of 2D- and 3D-evaluation, 2-grade analysis}

For the 2-grade classification, the agreement of 2D and 3D evaluation at the first reading ranged from 65 to $89 \%$. At the second reading, an agreement of $65-84 \%$ was ascertained. The evaluation of agreement according to Cohens-Kappa statistics showed mean $\kappa=0.45$ ( $\mathrm{SD} \pm 0.23$ ) for the first and $\kappa=0.45(\mathrm{SD} \pm 0.16)$ for the second reading (Table 2$)$.

\section{Interobserver agreement, 4-grade analysis}

The overall Interobserver agreement in the analysis of the 4-grade classification was $\kappa=0.48$ for $2 \mathrm{D}$ and $\kappa=0.53$ for $3 \mathrm{D}$ evaluation (Table 3 ).

\section{Interobserver agreement, 2-grade analysis}

The overall Interobserver agreement in the analysis of the 2 -grade classification was $\kappa=0.46$ for $2 \mathrm{D}$ and $\kappa=0.59$ for $3 \mathrm{D}$ evaluation (Table 3 ).

\section{Discussion}

The most important finding of this study is that data of interobserver reliability show better agreement values in the assessment of the 3D models compared to conventional MRI images, both, in the 4-degree as well as in the 2-degree classification. Although an absolute comparison of the Fleiss kappa values in the statistical analysis is not tenable due to different statistical assumptions, the comparison of the agreement values shows a slight superiority of the 
Table 3 Interobserver agreement, 2-grade- and 4 -grade analysis $* * *$

\begin{tabular}{|c|c|c|c|c|c|c|}
\hline & \multicolumn{3}{|l|}{$2 \mathrm{D}$} & \multicolumn{3}{|l|}{$3 \mathrm{D}$} \\
\hline & All & $\mathrm{t} 1$ & $\mathrm{t} 2$ & All & $\mathrm{t} 1$ & $\mathrm{t} 2$ \\
\hline 4-grade (Dejour) & 0.48 & 0.44 & 0.48 & 0.53 & 0.58 & 0.47 \\
\hline 2-grade (High/Low) & 0.46 & 0.43 & 0.40 & 0.59 & 0.63 & 0.57 \\
\hline
\end{tabular}

**** Fleiss-Kappa values of Interrater agreement; 4-grade, Dejour classification; 2-grade, high/low grade trochlear dysplasia; 2D, 2D axial MRI scan; 3D, MRI-based 3D model; $\mathrm{t} 1$, time point 1; 2 , time point 2
3D representation in the interobserver agreement. Further findings were that the analysis of intraobserver reliability showed no relevant differences between 2 and 3D representation, but better agreement values were found in the 2-degree classification compared to the 4-degree classification.

Trochlear morphology is a highly relevant parameter which is frequently discussed in international literature as it represents an important pathologic articular morphology that is a relevant risk factor for patellofemoral instability[2, $3,6,11,12,14-17,21]$.

One of the first studies using 3D imaging of the dysplastic trochlea was published by Biedert et al.[2]. In their study the authors showed that MRI 3D imaging was not only feasible, but they were able to identify variations in the dysplastic trochlea that were poorly represented using standard radiographs, CT scans, or routine 2D MRI imaging[2].

Fritz et al. postulated a higher proportion of correctly diagnosed cases of trochlear dysplasia after evaluation of 3D-printed models in comparison to $\mathrm{CR} / \mathrm{CT}$, and therefore, concluded that 3D models of the knee have the potential to improve diagnosis of patellofemoral dysplasia especially for less experienced surgeons [7]. The superiority of 3D representations has also been proven in other areas of medical practice. Wong et al. showed that 3D models of the hip joint can be beneficial for preoperative planning of femoroacetabular impingement surgery [29]. Another study demonstrated that 3D-printed models can precisely represent the size and shape of visceral aneurysms [23].

Apart from the analysis of the classification of trochlear dysplasia in 2D and 3D, another focus of this study was the distinction with regard to different classification options. In summary, the analysis carried out within this study shows a clear superiority of the 2-grade compared to the 4-gradeclassification by Dejour.

Unsatisfactory results in the agreement of Dejour's 4-grade classification were already reported in different publications [12, 21, 25, 28], why other classification-options presented in order to achieve more homogeneous results in the assessment of patellofemoral pathologies.

Biedert et al. [3] proposed significantly different trochlear medial and central condylar heights in patients with trochlear dysplasia [3].

Sharma et al. [21] developed a new classification system to assess the severity of trochlear dysplasia in axial MRI slices and demonstrated fair-to-good interobserver and goodto-excellent intraobserver agreement values, which, according to their classification, were found to be better than the Dejour classification on both CT and MRI [21].

Although interobserver and intraobserver agreements of other classification systems seem to be higher, Dejour's classification can still be regarded as state of the art when evaluating trochlear dysplasia [9]. On the basis of the results obtained within this study as well as the studies of the current literature, however, the Dejour classification as basis for a therapy decision must be questioned. With the development, application and validation of software-generated 3D models, as used within this study, the development of new 3D-based classification systems for the assessment of patellofemoral pathologies should also be forced.

This study has several limitations. First, the number of raters was relatively small with no radiologists included. Second, the number of included MRI scans was relatively small and no MRI scans of patients without trochlear dysplasia were analyzed. Third, no learning effect was investigated as this was the first-time use of the developed 3D models for assessing the patellofemoral anatomy. Despite the above-mentioned limitations, in view of the complex anatomy and the data obtained, the $3 \mathrm{D}$ representation for assessing the patellofemoral anatomy can be regarded as beneficial with regard to the reliability in classification of trochlear dysplasia and so potentially eases an automated analysis of the present pathology as well as an individualized surgical planning that is aimed in the future. Further, a 3D-based classification system potentially would befit the superiority, demonstrated in this study, of 3D representation in the assessment of trochlear dysplasia.

\section{Conclusions}

Overall, moderate-to-good agreement values were found in all groups. The analysis of intraobserver reliability showed no relevant differences between 2 and 3D representation, but better agreement values were found in the 2-degree classification compared to the 4-degree classification. With regard to the interobserver reliability, better agreement values were found in the $3 \mathrm{D}$ compared to the $2 \mathrm{D}$ representation. Therefore, in view data obtained within this study, the 
3D representation for assessing the patellofemoral anatomy can be regarded as beneficial with regard to the reliability in classification of trochlear dysplasia, which is relevant for future analytical procedures as well as surgical planning.

Author contributions $\mathrm{AF}$ and $\mathrm{KI}$ designed the study, collected data, performed the statistical analysis and wrote the manuscript. AF drafted the manuscript. AF, MJF, JD and JF assessed all MRI scans for trochlear dysplasia. MJF, JD and JF helped to design the study, assisted with statistical analysis and data interpretation, and critically reviewed the manuscript. MS, TY and HS helped with data interpretation and critically reviewed the manuscript. All authors read and approved the final manuscript.

Funding Open Access funding enabled and organized by Projekt DEAL. Contract grant sponsor: Helene-Lange Stiftung, Berlin. Contract grant sponsor: Deutsche Forschungsgemeinschaft (DFG, German Research Foundation); contract grant number: IZ 70 /2-1.

Availability of data and materials All relevant data are provided within the manuscript. The datasets used and/or analyzed during the current study are available from the corresponding author on reasonable request.

\section{Declarations}

Conflict of interest The authors declare that they have no conflict of interest with regard to the present study.

Ethical approval The study was approved by the institutional review board of the Technical University Munich and the study was performed in accordance with the Declaration of Helsinki.

Open Access This article is licensed under a Creative Commons Attribution 4.0 International License, which permits use, sharing, adaptation, distribution and reproduction in any medium or format, as long as you give appropriate credit to the original author(s) and the source, provide a link to the Creative Commons licence, and indicate if changes were made. The images or other third party material in this article are included in the article's Creative Commons licence, unless indicated otherwise in a credit line to the material. If material is not included in the article's Creative Commons licence and your intended use is not permitted by statutory regulation or exceeds the permitted use, you will need to obtain permission directly from the copyright holder. To view a copy of this licence, visit http://creativecommons.org/licenses/by/4.0/.

\section{References}

1. Balcarek P, Ammon J, Frosch S, Walde TA, Schuttrumpf JP, Ferlemann KG et al (2010) Magnetic resonance imaging characteristics of the medial patellofemoral ligament lesion in acute lateral patellar dislocations considering trochlear dysplasia, patella alta, and tibial tuberosity-trochlear groove distance. Arthroscopy 26:926-935

2. Biedert R, Sigg A, Gal I, Gerber H (2011) 3D representation of the surface topography of normal and dysplastic trochlea using MRI. Knee 18:340-346
3. Biedert RM, Bachmann M (2009) Anterior-posterior trochlear measurements of normal and dysplastic trochlea by axial magnetic resonance imaging. Knee Surg Sports Traumatol Arthrosc 17:1225-1230

4. Cusmano F, Adravanti P, Pedrazzini M, Ampollini A, Pavone P (2001) Radiologic assessment of femoro-patellar instability. Personal experience and review of the literature. Radiol Med 101:66-74

5. Dejour D, Le Coultre B (2007) Osteotomies in patello-femoral instabilities. Sports Med Arthrosc Rev 15:39-46

6. Dejour H, Walch G, Nove-Josserand L, Guier C (1994) Factors of patellar instability: an anatomic radiographic study. Knee Surg Sports Traumatol Arthrosc 2:19-26

7. Fritz B, Fucentese SF, Zimmermann SM, Tscholl PM, Sutter R, Pfirrmann CWA (2020) 3D-printed anatomic models of the knee for evaluation of patellofemoral dysplasia in comparison to standard radiographs and computed tomography. Eur J Radiol 127:109011

8. Keshmiri A, Schottle P, Peter C (2020) Trochlear dysplasia relates to medial femoral condyle hypoplasia: an MRI-based study. Arch Orthop Trauma Surg 140:155-160

9. Levy BJ, Tanaka MJ, Fulkerson JP (2021) Current concepts regarding patellofemoral trochlear dysplasia. Am J Sports Med 49:1642-1650

10. Lewallen LW, McIntosh AL, Dahm DL (2013) Predictors of recurrent instability after acute patellofemoral dislocation in pediatric and adolescent patients. Am J Sports Med 41:575-581

11. Li M, Ji G, Fan L, Fan CY, Lin W, Yang GM et al (2021) Assessment of patellar morphology in trochlear dysplasia on computed tomography scans. Orthop Surg 13:458-465

12. Lippacher S, Dejour D, Elsharkawi M, Dornacher D, Ring C, Dreyhaupt J et al (2012) Observer agreement on the Dejour trochlear dysplasia classification: a comparison of true lateral radiographs and axial magnetic resonance images. Am J Sports Med 40:837-843

13. Mousinho R, Ribeiro JNA, Pedrosa FKS, Lima DA, Goncalves RK, Leite JAD (2019) Evaluation of the reproducibility of the dejour classification for femoropatellar instability. Rev Bras Ortop (Sao Paulo) 54:171-177

14. Nelitz M, Lippacher S (2014) Arthroscopic evaluation of trochlear dysplasia as an aid in decision making for the treatment of patellofemoral instability. Knee Surg Sports Traumatol Arthrosc 22:2788-2794

15. Nelitz M, Lippacher S, Reichel H, Dornacher D (2014) Evaluation of trochlear dysplasia using MRI: correlation between the classification system of Dejour and objective parameters of trochlear dysplasia. Knee Surg Sports Traumatol Arthrosc 22:120-127

16. Pace JL, Cheng C, Joseph SM, Solomito MJ (2020) Effect of Trochlear dysplasia on commonly used radiographic parameters to assess patellar instability. Orthop J Sports Med 8:2325967120938760

17. Pfirrmann CW, Zanetti M, Romero J, Hodler J (2000) Femoral trochlear dysplasia: MR findings. Radiology 216:858-864

18. Redziniak DE, Diduch DR, Mihalko WM, Fulkerson JP, Novicoff WM, Sheibani-Rad S et al (2009) Patellar instability. J Bone Joint Surg Am 91:2264-2275

19. Remy F, Chantelot C, Fontaine C, Demondion X, Migaud H, Gougeon F (1998) Inter- and intraobserver reproducibility in radiographic diagnosis and classification of femoral trochlear dysplasia. Surg Radiol Anat 20:285-289

20. Salzmann GM, Weber TS, Spang JT, Imhoff AB, Schottle PB (2010) Comparison of native axial radiographs with axial MR imaging for determination of the trochlear morphology in patients with trochlear dysplasia. Arch Orthop Trauma Surg 130:335-340 
21. Sharma N, Brown A, Bouras T, Kuiper JH, Eldridge J, Barnett A (2020) The Oswestry-Bristol Classification. Bone Joint J 102B:102-107

22. Shen J, Qin L, Yao WW, Li M (2017) The significance of magnetic resonance imaging in severe femoral trochlear dysplasia assessment. Exp Ther Med 14:5438-5444

23. Shibata E, Takao H, Amemiya S, Ohtomo K (2017) 3D-printed visceral aneurysm models based on CT data for simulations of endovascular embolization: evaluation of size and shape accuracy. AJR Am J Roentgenol 209:243-247

24. Shoukri MM (2013) Measures of Interobserver Agreement and Reliability. Handbook of Inter-rater Reliability, 3rd Edition, Advanced Analytics, LLC, Gaithersburg; https://doi.org/10.1002/ 9781118445112.stat05301.pub22010:1-31

25. Smith TO, Davies L, Toms AP, Hing CB, Donell ST (2011) The reliability and validity of radiological assessment for patellar instability. A systematic review and meta-analysis. Skeletal Radiol 40:399-414

26. Steensen RN, Bentley JC, Trinh TQ, Backes JR, Wiltfong RE (2015) The prevalence and combined prevalences of anatomic factors associated with recurrent patellar dislocation: a magnetic resonance imaging study. Am J Sports Med 43:921-927

27. Stepanovich M, Bomar JD, Pennock AT (2016) Are the current classifications and radiographic measurements for trochlear dysplasia appropriate in the skeletally immature patient? Orthop J Sports Med 4:2325967116669490

28. Tscholl PM, Wanivenhaus F, Fucentese SF (2017) Conventional radiographs and magnetic resonance imaging for the analysis of trochlear dysplasia: the influence of selected levels on magnetic resonance imaging. Am J Sports Med 45:1059-1065

29. Wong TT, Lynch TS, Popkin CA, Kazam JK (2018) Preoperative use of a 3D printed model for femoroacetabular impingement surgery and its effect on planned osteoplasty. AJR Am J Roentgenol 211:W116-W121

Publisher's Note Springer Nature remains neutral with regard to jurisdictional claims in published maps and institutional affiliations.

\section{Authors and Affiliations}

\section{Andreas Fuchs ${ }^{1}$ (D) Matthias J. Feucht ${ }^{2} \cdot$ Jörg Dickschas ${ }^{3}$. Jannik Frings ${ }^{4} \cdot$ Markus Siegel $^{1} \cdot$ Tayfun Yilmaz $^{1}$. Hagen Schmal ${ }^{1} \cdot$ Kaywan Izadpanah $^{1}$}

\author{
Matthias J. Feucht \\ matthias.feucht@gmx.net \\ Jörg Dickschas \\ jdickschas@web.de \\ Jannik Frings \\ j.frings@uke.de \\ Markus Siegel \\ markus.siegel@uniklinik-freiburg.de \\ Tayfun Yilmaz \\ tayfun.yilmaz@uniklinik-freiburg.de \\ Hagen Schmal \\ hagen.schmal@uniklinik-freiburg.de
}

Kaywan Izadpanah

kaywan.izadpanah@uniklinik-freiburg.de

1 Department of Orthopedic Surgery and Traumatology, Freiburg University Hospital, Albert Ludwigs University Freiburg, Hugstetter Straße 55, 79106 Freiburg, Germany

2 Orthopädische Klinik Paulinenhilfe, Diakonieklinikum Stuttgart, Rosenbergstr. 38, 70176 Stuttgart, Germany

3 Klinik Für Orthopädie Und Unfallchirurgie, Klinikum Bamberg, Buger Strasse 80, 96049 Bamberg, Germany

4 Department of Trauma and Orthopaedic Surgery, University Medical Center Hamburg-Eppendorf, Martinistraße 52, 20246 Hamburg, Germany 